\title{
Reactive Element Effects in High-Temperature Alloys Disentangled
}

\author{
Vedad Babic $^{1} \mathbb{D} \cdot$ Christine Geers $^{1} \cdot$ Itai Panas ${ }^{1}$
}

Received: 19 July 2019 / Revised: 23 October 2019 / Published online: 23 November 2019

(C) The Author(s) 2019

\begin{abstract}
Reactive elements-REs - are decisive for the longevity of high-temperature alloys. This work joins several previous efforts to disentangle various RE effects in order to explain apparently contradicting experimental observations in alumina forming alloys. At 800-1000 ${ }^{\circ} \mathrm{C}$, "messy" aluminum oxy-hydroxy-hydride transients initially formed due to oxidation by $\mathrm{H}_{2} \mathrm{O}$ which in turn undergo secondary oxidation by $\mathrm{O}_{2}$. The formation of the transient oxide becomes supported by dispersed RE oxide particles acting as water equivalents. At higher temperatures, electron conductivity in impurity states owing to oxygen vacancies in grain boundaries (GBs) becomes increasingly relevant. These channels are subsequently closed by REs pinning the said vacancies. The universality of the emerging understanding is supported by a comparative first-principles study by means of density functional theory addressing $\mathrm{RE}(\mathrm{III}): \mathrm{Sc}_{2} \mathrm{O}_{3}, \mathrm{Y}_{2} \mathrm{O}_{3}$, and $\mathrm{La}_{2} \mathrm{O}_{3}$, and $\mathrm{RE}(\mathrm{IV}): \mathrm{TiO}_{2}, \mathrm{ZrO}_{2}$, and $\mathrm{HfO}_{2}$, that upon reaction with water, co-decorate a generic GB model by hydroxide and RE ions. At $100 \%$ RE coverage, the GB model becomes relevant at both temperature regimes. Based on reaction enthalpy $\Delta \mathrm{H}_{\mathrm{r}}$ considerations, "messy" aluminum oxy-hydroxyhydride transients are accessed in both classes. Larger variations in $\Delta \mathrm{H}_{\mathrm{r}}$ are found for RE(III)-decorated alumina GBs as compared to RE(IV). For RE(III), correlation with GB width is found, increasing with increased ionic radius. Similarly, upon varying RE(IV), minor changes in stability correlate with minor structural variations. GB decorations by $\mathrm{Ce}$ (III) and $\mathrm{Ce}$ (IV) further consolidate the emerging understanding. The findings are used to discuss experimental observations that include impact of co-doping by RE(III) and RE(IV).
\end{abstract}

Keywords Density functional theory $\cdot$ Reactive element effects $\cdot$ High-temperature oxidation · Alumina formers · Grain boundary decorations $\cdot$ Temperature effects

Vedad Babic

vedadb@chalmers.se

1 Department of Chemistry and Chemical Engineering, Chalmers University of Technology, 41296 Gothenburg, Sweden 


\section{Introduction}

Durability of functional alloys that serve at high temperatures relies on the formation of a protective slow-growing oxide scale to avoid breakaway oxidation. Aluminum and chromium constitute the main scale-forming elements in the commonly employed iron-, cobalt-, or nickel-base alloys. Greater thermodynamic stabilities of $\mathrm{Al}_{2} \mathrm{O}_{3}$ and $\mathrm{Cr}_{2} \mathrm{O}_{3}$ as compared to the oxides of the more noble base metal render both to form and enrich at the alloy surface, thereby providing the base metal protection from the harsh environment. When both $\mathrm{Al}$ and $\mathrm{Cr}$ are present in the alloy, $\mathrm{Cr}$ acts as 3rd element in that under oxidizing conditions, transient $\mathrm{Cr}_{2} \mathrm{O}_{3}$ inhibits internal oxidation of aluminum and facilitates the formation of the more stable alumina [1-3]. The 3rd element effect is most effective at intermediate temperatures, i.e., $800-1000{ }^{\circ} \mathrm{C}$. Indeed, due to kinetic considerations as well as absence of competitive structures to corundum, the formation of chromia is understood to act template for $\alpha-\mathrm{Al}_{2} \mathrm{O}_{3}$. At very high temperatures, i.e., higher than $1200{ }^{\circ} \mathrm{C}, \alpha-\mathrm{Al}_{2} \mathrm{O}_{3}$ becomes increasingly more readily accessed.

The electrochemical processes involved in scale growth include among others electron conduction. Here, the alloy/oxide interface is acting anode from which electrons are supplied to the outer oxide surface where the cathodic $\mathrm{O}_{2}$ reduction reaction takes place [4]. Outward diffusion of metal cations and inward diffusion of oxygen anions ensure the positive charge of the anodic interface and corresponding negative charge at the outer cathodic oxide surface to be kept at steady-state. The outward diffusion of cations is unwanted as it renders the receding alloy/oxide interface prone to void formation, which in turn compromises scale adherence. This is one of the effects that are countered by small additions (less than $1 \mathrm{wt} \%$ ) of socalled reactive elements REs to the alloy, e.g., Y, Zr, Hf, and Ce. The enigmatic roles that these additives, sometimes termed magic dust $[3,5,6]$, have been the topic of studies ever since the beneficial effects were first reported some 80 years ago; see [7]. Some 30 years ago, it was concluded that among these effects, mitigation of alumina scale spalling by improving scale/alloy adhesion was separable from the rest $[8,9]$.

The quest for understanding of the RE effect in alumina formers, first championed by Stringer, was consolidated and extended in studies by Pint, Heuer, and others. Four beneficial roles of reactive element additions have been formulated:

1. Elemental RE dopants are crucial inside the alloy where they offer sinks, e.g., the sulfur, carbon, nitrogen, and oxygen, that have remained in the matrix from the manufacturing stages. Under cyclic thermal conditions, sulfur, in particular, is known to enrich at the oxide/alloy interface where it impairs scale adhesion [3, 8-13].

2. At the alloy/oxide interface, elemental RE dopants become oxidized ending up decorating the oxide grain boundaries. This way, inhibition of grains coarsening is achieved, which in turn supports parabolic scale growth, i.e., suppresses the subparabolic kinetics owing to loss of grain boundary density [14, 15]. This is 
beneficial, in particular, at lower temperatures as it accelerates protective alumina scale growth [16-24].

3. RE doping achieves suppression of outward diffusion of $\mathrm{Al}^{3+}$ ions, thereby producing a well-adherent oxide scale. To explain this effect, a dynamic-segregation mechanism has been proposed, replacing outward diffusion of $\mathrm{Al}^{3+}$ by that of RE ions as driven by the oxygen activity gradient across the scale [6, 11, 23, 25-30].

4. The impact of RE doping was revisited by Heuer et al. [31, 32]. Thermal growth of alumina scale was subject to a fully electrochemical contextual analysis, including electronic conductivity properties as well as oxygen-activity-dependent ionic transport with bearing on the $\mathrm{RE}$ effect. In particular, $\mathrm{Y}_{2} \mathrm{O}_{3}$-attenuated electron conductivity was inferred based on inspirational studies of $\Sigma 7$ grain boundaries by means of first-principles density functional theory [32]. In a subsequent study, the $\Sigma 7$ grain boundary was subject to extended in-depth analysis by means of modeling [33]. Building on the latter study, but notwithstanding that high temperatures increasingly disfavor cooperative processes, their emerging working hypothesis regarding the RE effect still envisioned the moderation of cooperative migration at high temperatures, mainly as jogs along grain boundary disconnections, but this time also together with other conceivable cooperative mechanisms, the possible viability of any or all to be assessed by atomistic simulations [34].

And still, elemental additions of RE in the alloy must be kept low owing to unwanted excessive internal oxidation of RE inside the alloy or due to pegging [35]. In both cases, the oxygen activity originates from the dissociation pressure of alumina at the alloy/oxide interface.

Besides RE additions in the alloy in elemental form, RE oxide dispersions are common additives in oxide-dispersion-strengthened ODS alloys. At first, somewhat surprisingly, the impact of the RE oxide additive on scale growth bears great similarity to that of the elemental RE additives. However, in as much as elemental RE (e.g., Y, La, Zr, Hf) are very strong reducing agents, it readily follows that the oxidizing environment will render these elemental additives oxidized. To this end, the present study is part of a comprehensive effort that focuses on the impact on oxide scaling that any RE oxide particle residing at the outer surface of the component may have. Thereby, a complementary origin for the observed transient $\mathrm{RE}$ decoration of alumina grain boundaries is arrived at.

As longstanding as the roles of RE in oxidation of alumina formers, is the impact of water in high-temperature oxidation. Crucial effects include the incorporation of hydrogen in ionic form in the oxide, rendering it defect rich, which in turn controls the transport properties during scale growth [36]. Moreover, it was stated in [37] that even very dry gas mixtures-achieved, e.g., by employing phosphorous (V) oxide as drying agent-contain $\sim 3$ ppm $\mathrm{H}_{2} \mathrm{O}(\mathrm{g})$ which suffices to dominate or greatly influence the resulting defect structure in $\mathrm{Y}_{2} \mathrm{O}_{3}$ upon oxidation in nominally dry oxygen.

In general, conclusions as to what controls the formation of protective alumina, including RE effects, are based on scaling studies at high temperature. This is because as faster the oxidation is, the higher is the temperature, as scaling rates 
are often dominated by the activation energies for diffusions according to the Arrhenius equation. Thus, it was gratifying to unravel a crucial interplay of water and reactive elements at intermediate temperatures by combining experiment and modeling [38]. Oxide scale formation by water under nominally reducing and "dry" conditions was achieved in carefully controlled exposure experiments in $95 \% \mathrm{~N}_{2}, 5 \% \mathrm{H}_{2}, 35 \mathrm{ppm} \mathrm{H}_{2} \mathrm{O}$, and at $T=1173 \mathrm{~K}$ and $1273 \mathrm{~K}$. The nature of said oxide scale was subject to careful analysis. Rapid oxide scale formation on ODS FeCrAlY was found to involve two stages: (1) initial oxidation where water vapor comprises the only oxidant in conjunction with $\mathrm{Y}_{2} \mathrm{O}_{3}$ particles jointly supporting a rapidly growing transient nanogranular "messy" aluminum oxy-hydroxyhydride film and (2) a second stage where the transient oxide in turn becomes oxidized. Availability of yttria was found to control the local thickness of the transient "messy" oxide and contact with the notion of local overdoping/overdosing was also made. Preference for monodisperse submicron-sized yttria particles was advocated to avoid build up of stress and subsequent scale cracking owing to large local variations in scale thickness.

Driven by the oxidation of aluminum, in the first stage, the yttria additive facilitates water to, in effect, permeate the oxide scale. Hydrolysis followed by protonassisted inward diffusion of oxygen ions pins an inner cathode to the vicinity of the alloy/oxide interface where proton reduction takes place, being complementary to the alloy oxidation. Crucially, hydrogen is disposed as hydride ions in oxygen vacancies $\mathrm{H}^{-} @ \mathrm{~V}_{\mathrm{O}}^{* *}$ at an "inner cathode" in the vicinity of the mobile alloy/oxide interface. Thus, the transient defect-rich alumina nanograins formed become terminated jointly by $\mathrm{Y}^{3+}, \mathrm{OH}^{-}$and said $\mathrm{H}^{-} @ \mathrm{~V}_{\mathrm{O}}^{* *}$.

Subsequently, in the second stage, the resulting transient oxide film is understood to oxidize to finally produce a well adherent protective $\alpha-\mathrm{Al}_{2} \mathrm{O}_{3}$ scale. Yttrium enrichment is observed-in that yttrium aluminum garnet YAG nanograins form and grow in the upper oxide-simultaneously with the $\alpha-\mathrm{Al}_{2} \mathrm{O}_{3}$ formation and grains coarsening in vicinity of the alloy/oxide interface; see Scheme 1 . This outward diffusion of $\mathrm{RE}$ ions replacing that of $\mathrm{Al}^{3+}$ is reminiscent of the dynamic-segregation mechanism proposed by Pint [29] for high temperatures.

Indeed, in as much as the oxide grain boundaries constitute rapid diffusion paths, the time evolution of grain boundary density change becomes decisive for the rate of scale growth $[14,15]$. A crucial role of the RE becomes to disallow early grains coarsening-by the $\mathrm{Y}^{3+}$ decoration of nanograins - the consequence of which is the observed initial high rate of scale growth. The inwards growing initial "messy" oxide-besides being virtually free of voids-becomes sufficiently thick to ensure its transformation into the requested slow-growing protective $\alpha-\mathrm{Al}_{2} \mathrm{O}_{3}$ scale by the secondary oxidation step, and this despite low $\mathrm{Al}$ content in the alloy (5 wt\%).

This mechanism in two stages-rapid initial incomplete oxidation by $\mathrm{H}_{2} \mathrm{O}(\mathrm{g})+\mathrm{Y}_{2} \mathrm{O}_{3}$ followed by an oxidation step to achieve a well adherent oxide scale-was validated by combining experiments and first-principles modeling. Building on this mechanistic understanding and modeling procedure, the purpose of the present study is twofold, in that (1) it explores the degree of universality of the concerted mechanism by yttria being replaced by other RE oxides, and (2) generic insights are sought for how reactive elements may regulate the outward diffusion 


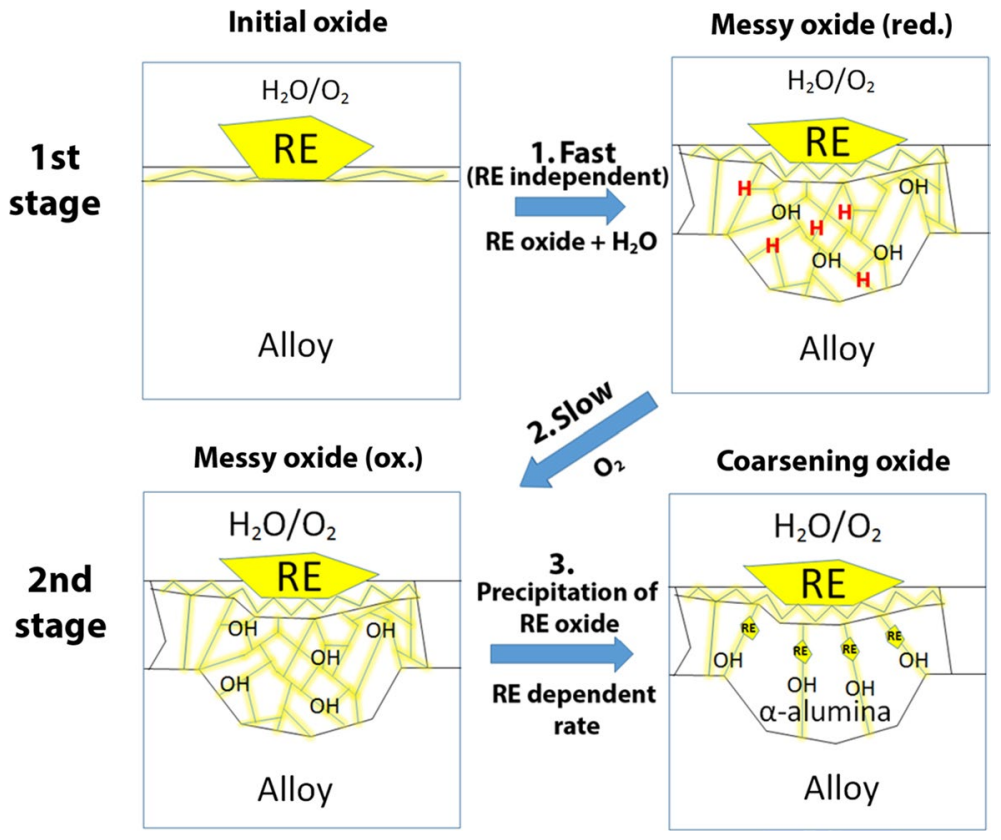

Scheme 1 Reactive element-assisted scale formation at intermediate temperatures disentangled: 1. Fast formation of a nanogranular messy oxide owing to aluminum oxidation by water. RE oxide assisting in scale permeation of water, in the form of $\mathrm{H}^{+}$and $\mathrm{OH}^{-}$, results in $\mathrm{RE}$ ions co-decoration of alumina grain boundary interfaces. Residual hydrogen, being accumulated as hydride ions in charged oxygen vacancies, renders the messy oxide partially reduced. 2. The partially reduced messy oxide undergoes oxidation by $\mathrm{O}_{2}$, rendering the resulting RE-decorated aluminum oxy-hydroxide unstable. 3. RE dependent rate of reprecipitation controls the rate of grains coarsening. The dynamic blocking effect combines 2 and 3 as the cathode process is taken to drive the outward diffusion of RE cations

of cations by RE ionic charges and radii, thereby causing time-dependent structural modifications in the grain boundaries.

Inspirational in our effort to unravel the crucial cooperation between water and RE at the early stages of oxidation of alumina formers is a similar phenomenology for ODS FeCrAlY [38] and ODS CoCrAlY [39]. This search for generic mechanistic insight from first principles benefits from maximum control of the model at hand. That, in turn, allows for systematic considerations in an ideal manner, here, both with regard to RE and composition of the model interface between alumina grains. Yet, the relevance of the emerging understanding - to real applications-must be established by means of experiments.

The present study is based on the properties of electroneutral monolayers of $\mathrm{RE}$ ions and hydroxides co-decorating interfaces between $\alpha-\mathrm{Al}_{2} \mathrm{O}_{3}$ slabs, cf. [38]. It provides energy landscapes as well as structural descriptors that emerge from systematically varying the monolayer compositions. The reactive elements considered comprise $\mathrm{Al}^{3+}, \mathrm{Sc}^{3+}, \mathrm{Y}^{3+}, \mathrm{La}^{3+}$ and $\mathrm{Ti}^{4+}, \mathrm{Zr}^{4+}, \mathrm{Hf}^{4+}$. These are classified according to oxidation numbers. Also, relevance of $\mathrm{Ce}^{3+}$ and $\mathrm{Ce}^{4+}$ in this context is explored. Contact between scale growth at intermediate and high temperatures 
is inferred in case of completely dehydrated grains boundaries, i.e., corresponding to $100 \%$ RE decorations.

\section{Modeling Considerations}

The density functional theory DFT based modeling approach described in [38] is reiterated here. Thus, steady-state yttria-assisted permeation of the alumina scale by water to achieve overall oxidation of aluminum at the alloy/oxide interface was validated employing a hydroxylated alumina model interface between 1.5-nm-thick lamella with a variable degree of co-decoration with $\mathrm{Y}^{3+}$ as mediating transients. Here, that understanding is generalized as two sets of RE are considered, having oxidation states III and IV, i.e., $\mathrm{RE}(\mathrm{III}): \mathrm{Al}^{3+}, \mathrm{Sc}^{3+}, \mathrm{Y}^{3+}, \mathrm{La}^{3+}, \mathrm{Ce}^{3+}$ and $\mathrm{RE}(\mathrm{IV}): \mathrm{Ti}^{4+}$, $\mathrm{Zr}^{4+}, \mathrm{Hf}^{4+}$, and $\mathrm{Ce}^{4+}$.

Caution is commonly warranted when employing present day's implementations of DFT to systems that may possess accessible local and delocalized Kohn-Sham states. This is owing to the self-interaction error SIE in DFT that favors the latter over the former. This commonly renders band gaps underestimated as valence bands are generally more localized than the conduction bands. Here, this is emphasized for Ce(III) owing to the small size of the occupied $4 f$ orbital. Consequently, any semiconductor properties associated with $\mathrm{Ce}$ (III) are disfavored as compared to the erroneous semi-metal. However, the electron density of said semimetallic states approximate the nominal VB states well, and thus the external potential, as seen by comparing computed crystal structure to the experimental for $\mathrm{Ce}_{2} \mathrm{O}_{3}$. Having said this, still the total energy of the faulty semimetallic $4 f$ band suffers from the SIE. In as much as the occupation of the $4 f$ states does not change during the chemical reactions considered, the impact of the SIE on the reaction energies approximately cancels. In the present study, this condition is satisfied throughout. Hence, while the description of $\mathrm{Ce}$ (III) comes with greater uncertainty than any of the other $\mathrm{RE}(\mathrm{III})$ and RE(IV) ions considered, it is deemed sufficient in the present classification study.

In the present study $\mathrm{Al}(\mathrm{s}), \mathrm{RE}(\mathrm{III})$ or $\mathrm{RE}(\mathrm{IV})$ oxides and a fully hydroxylated interface (see Fig. 1) between nanogranular alumina were taken as reactants. These are described by a generic periodic slab model that employs hydroxylated interfaces between $1.5 \mathrm{~nm}$ thick $\alpha-\mathrm{Al}_{2} \mathrm{O}_{3}$ lamella. The following reactions were considered for $\mathrm{RE}(\mathrm{III})$ and $\mathrm{RE}(\mathrm{IV})$, respectively:

$$
\begin{aligned}
& \frac{x}{2} \mathrm{RE}(\mathrm{III})_{2} \mathrm{O}_{3}(s)+x \mathrm{Al}(s)+\left(" \mathrm{H}_{2} \mathrm{O} "\right)_{12} @ \mathrm{~GB} \\
& \quad \rightarrow \frac{x}{2} \mathrm{Al}_{2} \mathrm{O}_{3}(s)+\left[\mathrm{RE}(\mathrm{III})_{2} \mathrm{O}_{3}\right]_{\frac{x}{2}}\left({ }^{\prime} \mathrm{H}_{2} \mathrm{O} "\right)_{12-\frac{3 x}{2}} @ \mathrm{~GB}+\frac{3 x}{2} \mathrm{H}_{2}(g) \\
& x=1,2, \ldots 8 .
\end{aligned}
$$




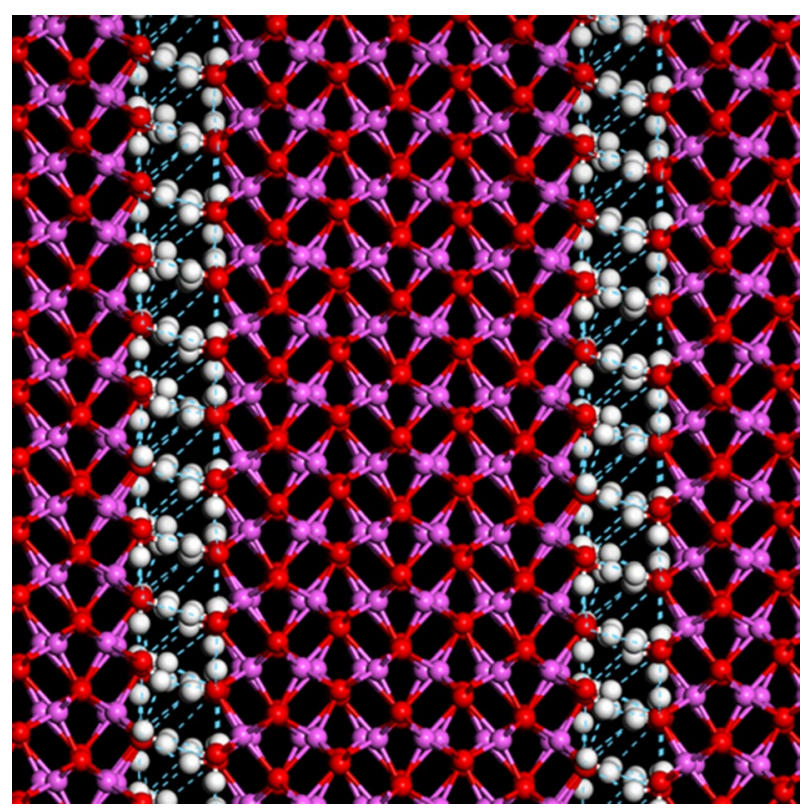

Fig. 1 Hydroxylated interface model between 1.5-nm-thick $\alpha-\mathrm{Al}_{2} \mathrm{O}_{3}$ lamella. Color code: $\mathrm{Al}$-purple; $\mathrm{O}-$ red; $\mathrm{H}$ - white, light blue dashed lines denote hydrogen bonds (Color figure online)

$$
\begin{aligned}
& x \mathrm{RE}(\mathrm{IV}) \mathrm{O}_{2}(s)+\frac{4 x}{3} \mathrm{Al}(s)+\left(" \mathrm{H}_{2} \mathrm{O} "\right)_{12} @ \mathrm{~GB} \\
& \quad \rightarrow \frac{2 x}{3} \mathrm{Al}_{2} \mathrm{O}_{3}(s)+\left[\mathrm{RE}(\mathrm{IV}) \mathrm{O}_{2}\right]_{x}\left({ }^{\prime} \mathrm{H}_{2} \mathrm{O} "\right)_{12-2 x} @ \mathrm{~GB}+\frac{x}{3} V_{\mathrm{Al}} @ \mathrm{~GB}+2 x \mathrm{H}_{2}(g) \\
& x=1,2, \ldots 6 .
\end{aligned}
$$

In $\mathrm{R} 1$ and $\mathrm{R} 2$, " $\mathrm{H}_{2} \mathrm{O}$ ” $@ \mathrm{~GB}$ reflects the fact that the hydroxylated interface is owing to hydrolysis. In $\mathrm{R} 2, V_{\mathrm{Al}} @ \mathrm{~GB}$ refers to the $\mathrm{Al}$ vacancy which is generated for every triad of RE(IV) that is introduced. The obtained reactions energies were offset by the hydrolysis reactions that maintain the hydroxylated interfaces [16]. As to the fate of hydrogen, a lower bound to the exothermicity is assumed by employing formation of $\mathrm{H}_{2}(\mathrm{~g})$ where in fact $\mathrm{H}^{-} @ \mathrm{~V}_{\mathrm{O}} * *$, hydrogen pick-up in the alloy, or hydrogen being oxidized to form water are the relevant hydrogen sinks. The reaction energies were equated with reaction enthalpies throughout, as reactants and products vibration energies as well as corresponding entropy were taken to cancel. Lower bounds to the Gibbs free energy landscape for the codecoration of grain boundaries by $\mathrm{Y}^{3+}$, and hydroxides were estimated by taking the loss of translational entropy of water, $144 \mathrm{JK}^{-1} \mathrm{~mol}^{-1}\left(\sim 1.5 \mathrm{meVK}^{-1}\right)$ to comprise the only entropy change of the reaction. Said lower bound is because the increase in configurational entropy of the resulting mixed solid, i.e., RE-decorated nanogranular aluminum oxy-hydroxy-hydride "messy" scale is neglected. 
Thus, an estimated upper bound for the temperature where the co-decoration of $\mathrm{Y}^{3+}$ and hydroxides is spontaneous, i.e., $\Delta \mathrm{G} \leq 0$, may be formulated according to

$$
T \leq \frac{\Delta H}{\Delta S}
$$

Robustness of the understanding arrived at employing said hydroxylated interfaces between $1.5 \mathrm{~nm}$ thick alumina lamella co-decorated with $\mathrm{Y}^{3+}$, which was validated by removing the alumina scaffold all together employing a quasi-2D $\mathrm{Al}(\mathrm{III}) \mathrm{OOH}$ structure, based on Gibbsite, to model the hydroxylated interface.

The spin-polarized DFT calculations in this study were performed using the CASTEP code within the Materials studio 6.0 suite [40]. The PBE-GGA functional was employed for all calculations. Non-local Vanderbilt ultrasoft pseudopotentials have been used to describe core electrons together with a $550 \mathrm{eV}$ energy cutoff, and the Brillouin zone was sampled with $0.04 \AA^{-1}$ k-points separation employing a $3 \times 3 \times 2$ sampling mesh [41]. The convergence criteria included $10^{-3} \AA$ (displacement) and $10^{-5} \mathrm{eV} /$ atom (energy). Impact of London interaction [42] on the structure and energetics of co-decoration was previously found to be negligible for $\mathrm{Y}^{3+}$. Here, this was found to hold true also for $\mathrm{Zr}^{4+}$.

\section{Results}

Two sets of reactive elements RE(III) and RE(IV) were investigated, in order to assess the degree to which the emerging understanding regarding the yttria effect at intermediate temperatures, as reported in [38], is indeed universal. Conversion of $\mathrm{RE}$ oxide particles to $\mathrm{RE}$ ions decorating alumina grain boundaries driven by aluminum oxidation by water according to $\mathrm{R} 1$ and $\mathrm{R} 2$ is summarized in Fig. 2a, and is converted into corresponding Gibbs energy topographies. All reaction enthalpies in Fig. 2 a come out exothermic, originating from the high enthalpic drive of aluminum oxidation by water. The stability separation between $\mathrm{RE}$ (III) and RE(IV) bundles in Fig. 2a originates from the fact that $\frac{3}{2} \mathrm{H}_{2}$ evolves from $\frac{3}{2} \mathrm{H}_{2} \mathrm{O}$, while each $\mathrm{RE}(\mathrm{IV})$ is associated with the evolution of $2 \mathrm{H}_{2}$ from 2 $\mathrm{H}_{2} \mathrm{O}$. What truly differs between the $+\mathrm{III}$ and $+\mathrm{IV}$ bundles is the larger variation in reaction energies among the former set. This spread is consistent with correspondingly larger variations in grain boundary width as quantified by the $\mathrm{O}-\mathrm{O}$ distances across interfaces between alumina slabs; see Figs. 3 and 4 for + III and $+\mathrm{IV}$, respectively. It is observed that with the exception of $\mathrm{Ce}(\mathrm{IV})$, regardless of RE(IV) size, the grain boundary width remains virtually unchanged. Indeed, complexity in variations of GB widths as function of RE(III) concentration is intuitive, i.e., owing to varying degrees of compatibility of cation sizes with the coexisting structure former, comprising the hydroxides offering intergranular hydrogen bonding. The absence of such complexity in case of RE(IV) may at first be counterintuitive. It may partly be understood to originate from the condition of charge neutrality in the alumina scaffold enforcing every three RE(IV) residing in the interface to coexist with a nominal cationic vacancy. Thus when cation 

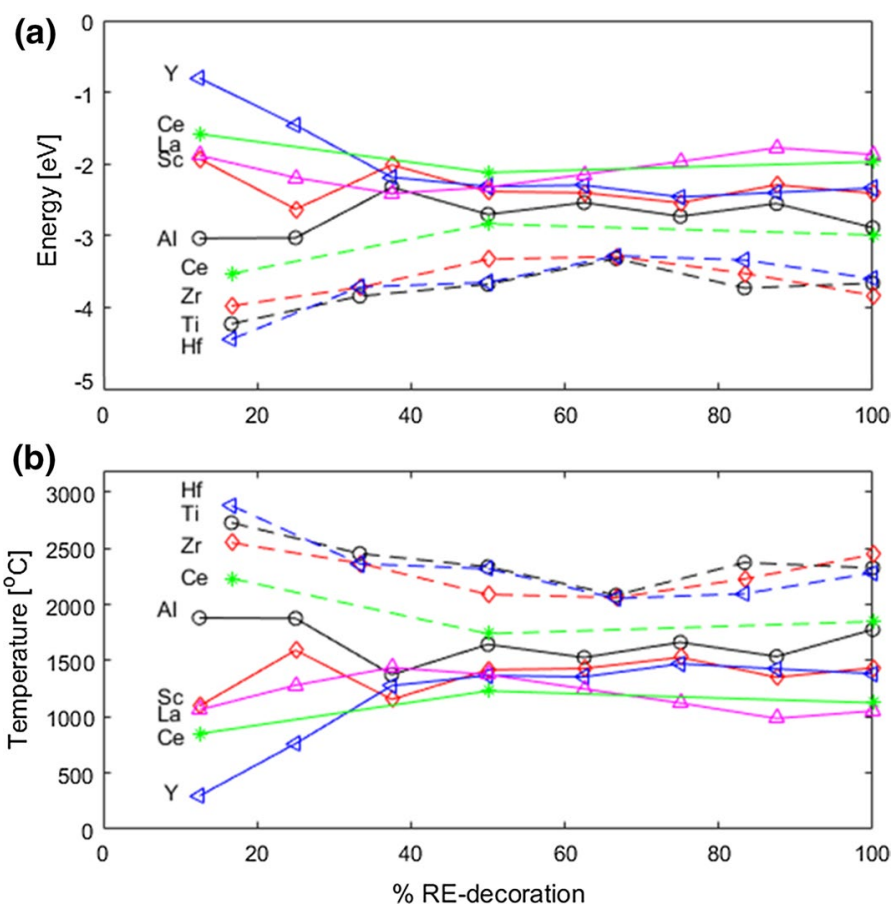

Fig. 2 a Reaction energies and b temperatures for the $\mathrm{RE} / \mathrm{H}_{2} \mathrm{O}$ co-decoration reaction, cf. R1 and R2. The temperature topographies determine the maximum temperatures for spontaneous formation for formation of RE-decorated grain boundaries from the corresponding RE oxides, see Fig. 1b. Overall, with the exception of $\mathrm{Ce}^{4+}$ (see more below), the +III (full lines) and +IV cations (dashed lines) are separated in bundles with the + III cations showing the larger variations in stability, especially at lower coverages

radius changes $\mathrm{R}\left(\mathrm{Ti}^{4+}\right)<\mathrm{R}\left(\mathrm{Zr}^{4+}\right) \approx \mathrm{R}\left(\mathrm{Hf}^{4+}\right)<\mathrm{R}\left(\mathrm{Ce}^{4+}\right)$ the corresponding buildup of stress may be relaxed by lateral deformations in case of the three former and thus no increase in $\mathrm{O}-\mathrm{O}$ distances across the grain boundary is needed. An exception to this rule is offered by $\mathrm{Ce}^{4+}$, the ionic radius of which is significantly larger than that of the other $\mathrm{RE}(\mathrm{IV})$ ions considered in that $\mathrm{R}\left(\mathrm{Ce}^{4+}\right) \approx \mathrm{R}\left(\mathrm{Y}^{3+}\right)$. Thus, it comes out intermediate between the two classes. Indeed, the inability to achieve lateral relaxation in case of $\mathrm{RE}(\mathrm{III})$ is reflected in the observed larger variations in GB widths related to $\mathrm{RE}(\mathrm{III})$ ion size dependence. The mean $\mathrm{O}-\mathrm{O}$ distances increase with increasing cation size $\left(\mathrm{R}\left(\mathrm{Al}^{3+}\right)<\mathrm{R}\left(\mathrm{Sc}^{3+}\right)<\mathrm{R}\left(\mathrm{Y}^{3+}\right)<\mathrm{R}\left(\mathrm{La}^{3+}\right) \approx \mathrm{R}(\mathrm{C}\right.$ $\mathrm{e}^{3+}$ ) becomes especially clear when comparing $\mathrm{Y}^{3+}$ and $\mathrm{La}^{3+}$. While the smaller RE(III) cations may be accomodated, the increasing structural mismatch of the larger RE(III) cations drives the separation between interface planes, consequently causing stresses owing to the GB decoration.

Besides interatomic distances across grain boundaries, the difference between $\mathrm{RE}(\mathrm{III})$ and RE(IV) decorations are manifested in connectivities among potentially viable interstitial sites for cation accommodation as well as mobility. Here, this is visualized for $100 \%$ coverage for a selected electrostatic potential 


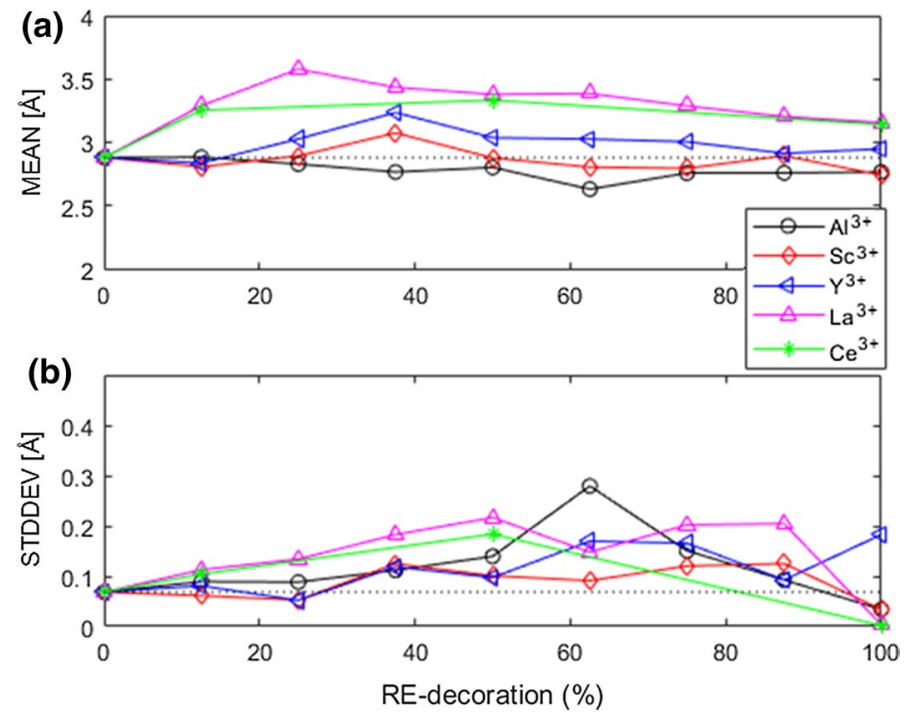

Fig. 3 Two properties related to the impact of $\mathrm{RE}$ (III) decoration on the effective grain boundary width measured by the mean oxygen-oxygen distance across the two interface planes. a Mean GB widths and b corresponding standard deviations of the local oxygen-oxygen distances are displayed as function of RE(III) coverage. Larger cations cause larger GB widths
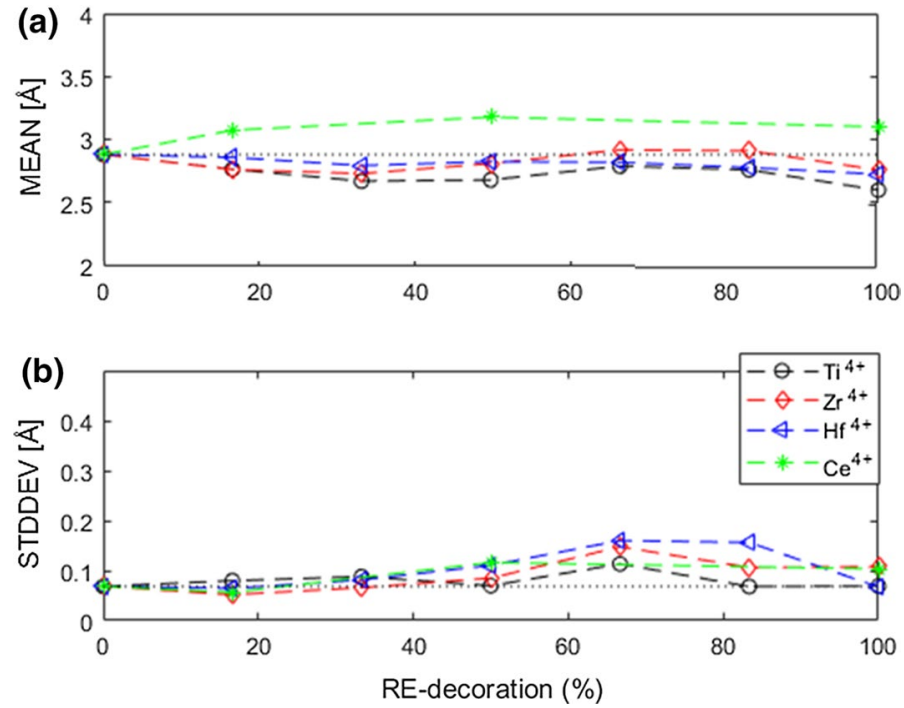

Fig. 4 Impact of $\mathrm{RE}(\mathrm{IV})$ decoration on the effective grain boundary width measured by the mean $\mathrm{O}-\mathrm{O}$ distance across the two interface planes. a Mean GB widths and $\mathbf{b}$ corresponding standard deviations of the local O-O distances are displayed as function or RE(IV) coverage. Note the minor variations in both graphs, the exception being $\mathrm{Ce}^{4+}$ and this owing to its significantly larger ionic radius 


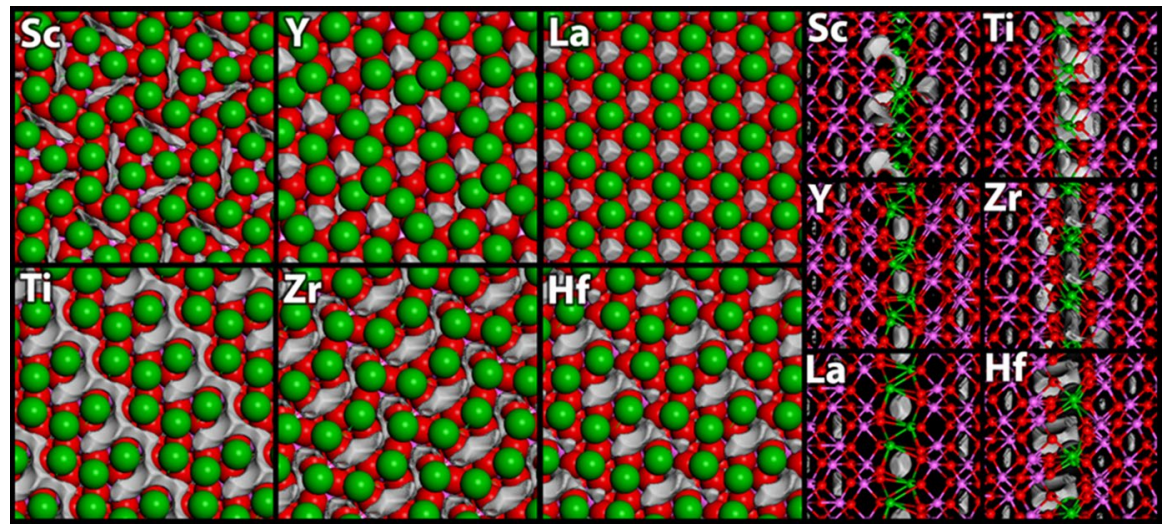

Fig. 5 Negative electrostatic potential isosurfaces EPI:s (gray) for the RE-decorated interfaces. Front view (left). Side view (right). Localized EPI:s for $\mathrm{RE}(\mathrm{III}) \mathrm{Sc}^{3+}<\mathrm{Y}^{3+} \sim \mathrm{La}^{3+}$, are contrasted by more extended EPI:s among RE(IV) $\mathrm{Ti}^{4+}>\mathrm{Zr}^{4+} \sim \mathrm{Hf}^{4+}$. (Red balls: Oxygen; green balls: RE; Purple balls: Aluminum) (Color figure online)

isosurface EPI, see Fig. 5. For the RE(III) ions, $\mathrm{Sc}^{3+}, \mathrm{Y}^{3+}, \mathrm{La}^{3+}$, little effect of ionic radius increase on the EPI is observed. The similarly disconnected EPI among RE(III) is consistent with structural changes owing to an increase in radius to be manifested as changes in the grain boundary widths. However, for the $\mathrm{RE}(\mathrm{IV})$ ions, i.e., $\mathrm{Ti}^{4+}, \mathrm{Zr}^{4+}, \mathrm{Hf}^{4+}$, the EPI goes from being interconnected in case of $\mathrm{Ti}^{4+}$ to increasingly fragmented in case of $\mathrm{Zr}^{4+}$ and $\mathrm{Hf}^{4+}$. It offers a further manifestation of lateral relaxations in the interface absorbing the impact of RE(IV) cation size increase.

\section{Discussion}

Strategies to achieve control of oxide scaling on alumina formers at high temperatures $>1200{ }^{\circ} \mathrm{C}$ by employing elemental RE dopants are described in the Introduction section. In addition, the interplay between water and RE oxide particles that reside on the outer surface of the specimen has been shown to impact the scaling in a major way at intermediate temperatures, i.e., $800-1000{ }^{\circ} \mathrm{C}$ [38], where electrochemistry is expected to be slow. The observed promotion is owing to yttria assisting in the oxidation process whereby the alloy becomes oxidized by water. At early stages, the observed yttria decoration results from the yttria acting "water equivalent" supporting the oxidation process, where the $\mathrm{Y}^{3+}$ ions end up decorating the resulting aluminum oxy-hydroxy-hydride nanograins of a "messy oxide" that grows exclusively inwards owing to the inner cathode; see Scheme 1. This generic process exhibits variations in stabilities mainly among RE(III) ions and at low RE coverages; see Fig. 2b. At subsequent stages, the Wagnerian oxidation channel owing to $\mathrm{O}_{2}$ reduction becomes increasingly more important, whereby the resulting RE-decorated aluminum oxy-hydroxy hydride nanograins become oxidized by $\mathrm{O}_{2}$ acting outer cathode. This secondary oxidation of the 
"messy" oxide renders nanograins to fuse and cause the RE cations in the grain boundaries to diffuse back into the outer oxide toward the outer cathode, reminiscent of the dynamic-segregation mechanism of Pint [29]. The fact that smaller RE cations do not block outwards diffusion of aluminum to the same extent as the larger cations have been attributed to the former partially dissolving in the alumina matrix, disallowed owing to ionic radius mismatch for the larger cations forcing these to reside in the grain boundaries. Indeed, during alumina grains coarsening in the inner part, precipitation of RE-rich oxide particles was observed in the outer part of the oxide scale [38]. As a consequence of the continuous loss of grain boundary density, the initial parabolic scale growth kinetics goes subparabolic. Arguably, the stability of the transient RE-decorated grain boundaries suppresses this transformation, thus rendering the rate of oxide growth greater for $\mathrm{RE}(\mathrm{IV})$ decoration than for $\mathrm{RE}$ (III) and greater for $\mathrm{RE}(\mathrm{III})$ decoration than in absence of RE decoration, cf. Figure 2. The overall change in scale thickness at intermediate temperatures $\sim 800-1000{ }^{\circ} \mathrm{C}$ was modeled in $[15,38]$, cf. Figure $6 \mathrm{a}$,

$$
X=\left\{2 \frac{D_{\mathrm{GB}} 2 \delta_{\mathrm{GB}}}{k} \frac{\Delta \mu}{R T} \ln \left[1+\frac{t}{t_{0}}\right]\right\}^{\frac{1}{2}},
$$

i.e., early parabolic $X \propto \sqrt{t}$, and late $X \propto \sqrt{\ln (t)}$. Note that in general the different dynamic-segregation effects of different RE additives affect the grain boundary diffusivity differently, thus causing the rates of mass gain to differ also in the parabolic regime, i.e., prior to grains coarsening.

This scaling provides the initial conditions for scale growth at higher temperatures in that said transformation reflects the further increased relevance of
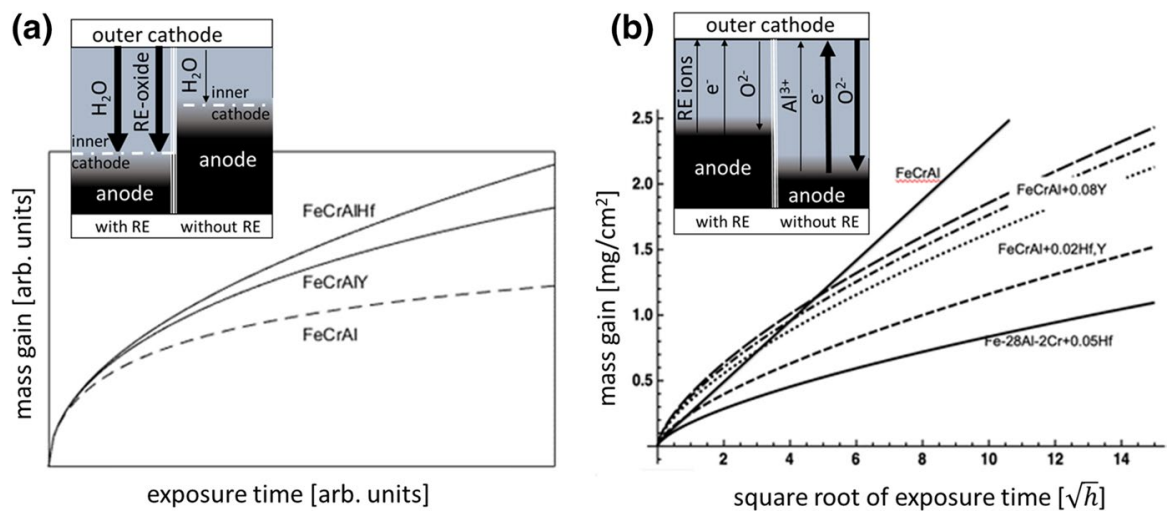

Fig. 6 Mass gain curves. a Modeling behavior at intermediate temperatures, cf. Equation 1. Three cases are illustrated: undoped (bottom), impact of $\mathrm{Y}_{2} \mathrm{O}_{3}$ OD (middle), and $\mathrm{HfO}_{2} \mathrm{OD}$ (top) in FeCrAl alloy. Undoped $\mathrm{FeCrAl}$ shows lowest mass gain owing to grains coarsening initiated very early in the absence of $\mathrm{RE}$ decoration of grain boundaries. It is followed by $\mathrm{Y}^{3+}\left(\right.$ or $\left.\mathrm{La}^{3+}\right)$ and $\mathrm{Hf}^{4+}$ (or $\mathrm{Zr}^{4+}$ ) decorated grain boundaries FeCrAl, i.e., in stability order (cf. Figures 2, 3, 4). b Modeling isothermal behavior at high temperatures, cf. Eq. 2, experiment is [23]. Linear is $\Delta m \propto \sqrt{t}$. Sublinear is $\Delta m \propto t^{\frac{1}{3}}$. Undoped FeCrAl shows parabolic scale growth, while RE decoration inhibits transport in grain boundaries. Insets illustrate the opposite impacts of RE on scale growth at $\mathbf{a}$ intermediate and $\mathbf{b}$ high temperatures 
the outer cathode, i.e., by $\mathrm{O}_{2}$ reduction. It is achieved by the enhanced electronic conductivity across the scale emerging from the top-of-valence-band to the bottom-of-conduction-band electronic excitations that in turn become facilitated by chemical disruptions in high-angle grain boundaries [32-34], and/or by employing oxygen vacancies as generic impurity states in the band gaps of the grain boundaries [2, 16, 38]. In [2], bipolaron-mediated redox processes among $V_{\mathrm{O}}$ sites were shown to drastically enhance $V_{\mathrm{O}}$ mobility and thus to facilitate thermal oxide growth. The change in scaling conditions is captured by the superparabolic-cubic model [15]; see Fig. 6b,

$$
X=\left(9 D_{\mathrm{GB}} 2 \delta_{\mathrm{GB}} t_{0}^{c} \frac{\Delta \mu}{R T}\right)^{\frac{1}{3}}\left[\sqrt{1+\frac{t}{t_{0}^{c}}}-1\right]^{\frac{2}{3}}
$$

Interestingly, Eq. 2 requires initial grain boundary density increase to reach a maximum at $t=\frac{5}{4} t_{0}^{c}$, prior to subsequent grains coarsening to occur. Indeed, the two asymptotic limits for Eq. 2 are the superparabolic $X \propto t^{\frac{2}{3}}$ for $t \ll t_{0}^{c}$ and the cubic $X \propto t^{\frac{1}{3}}$ for $t \gg t_{0}^{c}$, the latter independent of $t_{0}^{c}$.

On activation of the outer cathode at high temperatures, the dynamic-segregation mechanism [29] too is activated. The former is because electron hopping becomes increasingly accessible, while the latter infers that besides the inward diffusion of anions, the complementary outward diffusion of aluminum ions becomes replaced by that of RE ions [25, 28, 29]. The outward migration of RE is indeed reported again and again in the literature; see, e.g., [35]. A feedback effect of RE ions attenuating the electron conduction in turn is arrived at. Indeed, in the absence of RE, early parabolic scaling is observed, while cubic oxide growth is found upon RE doping. These observations support RE-induced oxidation slowdown by pinning impurity states in the band gap, cf. [2] and also [32-34]. Greater suppression by RE(IV) than RE(III) is consistent with greater stability in case of the former, cf. Figure 2. It is concluded that at intermediate temperatures, REs assist the oxidation when water acts oxidant. At elevated temperatures though, REs suppress the oxidation by $\mathrm{O}_{2}$ (i) by reducing electron conductivity and (ii) by suppressing the outward diffusion of aluminum ions. The latter renders the oxide scale inward growing owing to inward diffusion of oxygen ions, that in turn results in apparent grains coarsening as reflected in the cubic mass gain curves [23]; see Fig. 6b again. Subsequent reprecipitation of RE oxide particles promotes the further coarsening of alumina grains.

It is inferred from Figs. 2, 3, and 4 that reprecipitation of $\mathrm{Ce}^{4+}$ or RE(III) in the outer oxide is faster than for $\mathrm{Zr}^{4+}$ or $\mathrm{Hf}^{4+}$. This is owing to the buildup of additional stress in case of the former as manifested in the RE concentration dependence of the grain boundary width (see Figs. 3, 4). Indeed, a potentially crucial consequence is that the decay of grain boundary density becomes slower for $\mathrm{Zr}^{4+}$ and $\mathrm{Hf}^{4+}$ than for $\mathrm{Ce}^{4+}$ or $\mathrm{RE}(\mathrm{III})$. Thus, the water permeation channel is kept open longer than in case of both $\mathrm{RE}(\mathrm{III})$ and $\mathrm{Ce}^{4+}$ allowing for the "messy oxide" to grow thicker. Repeatedly, the mainly non-electrochemical channel sustains a well-adherent scale by maintaining an inner cathode in vicinity of the alloy/oxide interface. This renders obsolete the electrochemical requirement of compensating outwards diffusion of cations in order to maintain constant the steady-state charge separation across the 
oxide scale. It is noted here that would the RE source be elemental and reside in the alloy matrix, "pegs" would readily form owing to internal oxidation. In contrast, oxidation due to the interplay of water and RE oxide is terminated from below by the RE oxide grain size.

Said absence of grain boundary width driven formation of RE-rich particles in case of $\mathrm{Zr}^{4+}$ and $\mathrm{Hf}^{4+}$, while present in case of $\mathrm{RE}(\mathrm{III})$, as well as for $\mathrm{Ce}^{4+}$, offers the opportunity to optimize the scale adhesion property while maintaining a slowgrowing oxide. Thus, it is tempting to propose improved protective properties to result from co-doping alumina formers by $\mathrm{RE}(\mathrm{III})$ and $\mathrm{RE}(\mathrm{IV})$ oxide dispersions also in line with $[23,43]$. These authors found that all REs decorate grain boundaries independent of doped separately or being co-doped. Yet, oxide scale growth kinetics showed superior behavior by co-doping with RE(III) and RE(IV). First and foremost, it is the time evolution of the grain boundary density that is modified by tuning this mixture. In as much as scale growth benefits from suppression of alumina grains coarsening, this is achieved by $\mathrm{RE}(\mathrm{IV})$ decoration of grain boundaries. Interestingly, in locally mixed co-decorations of grain boundaries by $\mathrm{RE}(\mathrm{III})$ and $\mathrm{RE}(\mathrm{IV})$, the former may benefit from the nominal Al vacancies, see R2, generated by the RE(IV) ions, i.e., RE(III) decoration-induced stress owing to grain boundary width expansion is avoided. Thus, owing to zirconium or hafnium dopants, e.g., YAG formation and corresponding alumina grains coarsening is suppressed in favor of sustained grain boundary co-decoration of $\mathrm{Y}^{3+}$ and $\mathrm{Zr}^{4+}$ or $\mathrm{Hf}^{4+}$ (Fig. 2b). Also based on stability arguments, mixing different REs may indeed be beneficial. In as much as $\mathrm{La}(\mathrm{III}) @ \mathrm{~GB}$ or $\mathrm{Ce}(\mathrm{III}) @ \mathrm{~GB}$ forms more readily from their corresponding sesquioxides than does $\mathrm{Y} @ \mathrm{~GB}$, the former may assist the latter at low RE coverage, cf. Figure 2. While both $\mathrm{Ce}_{2} \mathrm{O}_{3}-\mathrm{Ce}$ (III)@GB and $\mathrm{CeO}_{2}-\mathrm{Ce}(\mathrm{IV}) @ \mathrm{~GB}$ couples have been addressed in the present study, it is reasonable to assume, based on thermodynamics as displayed in, e.g., the Ellingham diagram, that $\mathrm{Ce}_{2} \mathrm{O}_{3}-\mathrm{Ce}$ (III)@GB is the relevant one at early stages in the oxidation process. This is because-at a given $\mathrm{pO}_{2}$-oxidation of $\mathrm{Ce}(\mathrm{s})$ to form $\mathrm{Ce}_{2} \mathrm{O}_{3}(\mathrm{~s})$ precedes oxidation of $\mathrm{Al}(\mathrm{s})$ to form $\mathrm{Al}_{2} \mathrm{O}_{3}(\mathrm{~s})$ that in turn precedes the oxidation of $\mathrm{Ce}_{2} \mathrm{O}_{3}(\mathrm{~s})$ to $\mathrm{CeO}_{2}(\mathrm{~s})$. Finally, also based on thermodynamic considerations it is tempting to assume $\mathrm{Ce}$ (III)@GB to become oxidized by " $\mathrm{H}_{2} \mathrm{O}$ ” $@ \mathrm{~GB}[44,45]$. However, repeating the previous reasoning renders $\mathrm{Al}$ as the actual reducing agent and consequently the $\mathrm{Ce}_{2} \mathrm{O}_{3}-\mathrm{Ce}$ (III)@ GB couple prevails in grain boundaries that maintain "water permeation" to the alloy/oxide interface in case of cerium as reactive element.

\section{Summary and Conclusion}

Possible complementary reactive element effects in alumina formers at high and intermediate temperatures were described.

- At high temperatures, alumina scale formation was explained by conventional Wagner theory, i.e., outer cathode: reduction of $\mathrm{O}_{2}$ at the gas/oxide interface, anode: oxidation of metal at the alloy/oxide interface, albeit electron and ions 
transport across the scale are confined to oxide grain boundaries. The RE additives offer control by suppressing the GB-mediated transport.

- At intermediate temperatures, Wagner theory supports only very slow oxide growth by the outer cathode. Observed rapid oxidation is owing to RE oxide effectively facilitating water to permeate the scale to reach an inner cathode. RE oxide additives thus support scaling in that it aids the oxidation by water to build a sufficiently thick and well-adherent transient oxide scale.

The two properties become coupled in thermal cycling applications, e.g., jet turbines, where the conditions for transient oxide formation reappear in each cycle and therefore repeatedly set the stage for the high-temperature oxidation. An emerging class of applications concerns alumina formers as nuclear fuel cladding materials to achieve improve resilience also under accident conditions by utilizing the slower oxidation kinetics of FeCrAlRE alloys, i.e., by the formation of chromia under working conditions and increasingly alumina as temperature rises-as compared to conventional zirconium alloys [46]. Crucially, by safeguarding the RE effect at intermediate temperatures, scale spalling at high temperature is avoided.

Universality as to the interplay between reactive elements and water, see Scheme 1 and [38], was validated, in particular, for intermediate temperatures.

1. RE(III) causes significant increase in GB width with increased ionic radius, suggesting oxidation-driven buildup of stress to support rapid RE oxide reprecipitation and corresponding grains coarsening. Thus, rapid transition from parabolic to subparabolic scale growth kinetics is inferred.

2. $\mathrm{RE}(\mathrm{IV})$, with the exception of $\mathrm{Ce}^{4+}$, very small changes in GB widths are observed upon increasing RE ions radii. Owing to the greater stabilities, slower $\mathrm{RE}$ oxide reprecipitation is expected allowing more enduring parabolic scale growth kinetics

3. Thermodynamic considerations under steady-state conditions imply the Ce speciation to comprise the $\mathrm{Ce}_{2} \mathrm{O}_{3}-\mathrm{Ce}$ (III) @ GB couple - the former at the outer oxide interface and the latter in the grain boundaries-for as long as these effectively permeate water to the alloy/oxide interface where aluminum is oxidized.

4. It is inferred that mixing different RE oxide dispersions may provide enhanced control of initial "messy oxide" formation at intermediate temperatures. Indeed, in as much as $\mathrm{Y}$ (III)@ GB from $\mathrm{Y}_{2} \mathrm{O}_{3}$ comes out non-spontaneous above $500{ }^{\circ} \mathrm{C}$ at low coverage, this may indeed be compensated by both the $\mathrm{La}_{2} \mathrm{O}_{3}-\mathrm{La}$ (III)@ GB and the $\mathrm{Ce}_{2} \mathrm{O}_{3}-\mathrm{Ce}(\mathrm{III}) @ \mathrm{~GB}$ couples.

5. At later stages, the duration of parabolic scale growth preceding grains coarsening may be achieved by tuning the resilience of the grain boundary density by co-decoration of RE(III) and RE(IV), i.e., whereby the drive for secondary RE oxide reprecipitation becomes suppressed as RE(III) may utilize the vacancies generated by the RE(IV) to some extent alleviate the inherent stress associated with RE(III)@GB.

6. $\mathrm{RE}(\mathrm{IV})$ decoration of alumina grain boundaries enforces aluminum vacancies owing to the charge neutrality constraint enhancing RE(IV) mobility while at the 
same time suppressing $\mathrm{Al}^{3+}$ mobility whereby a dynamic-segregation effect is achieved.

Thus, alloys with improved corrosion resistance are anticipated from the fact that superior control of oxide scale growth kinetics may be achieved along two different routes: by the interplay between water and the RE oxide dispersion at intermediate temperatures and by elemental RE dopants in the alloy at high temperatures.

Acknowledgements Open access funding provided by Chalmers University of Technology. This work was carried out partly within the Swedish High Temperature Corrosion Center (CG and VB) and within the Swedish Foundation for Strategic Research project SAFETY (VB and IP). Computations were performed on resources at Chalmers Centre for Computational Science and Engineering (C3SE) provided by the Swedish National Infrastructure for Computing (SNIC).

Open Access This article is distributed under the terms of the Creative Commons Attribution 4.0 International License (http://creativecommons.org/licenses/by/4.0/), which permits unrestricted use, distribution, and reproduction in any medium, provided you give appropriate credit to the original author(s) and the source, provide a link to the Creative Commons license, and indicate if changes were made.

\section{References}

1. Z. G. Zhang, F. Gesmundo, P. Y. Hou and Y. Niu, Corrosion Science 48, 741 (2006).

2. V. Babic, C. Geers and I. Panas, RSC Advances 8, 41255 (2018).

3. D. P. Whittle and J. Stringer, Physical and Engineering Sciences 295, 309 (1980).

4. C. Wagner, Corrosion Science 5, 751 (1965).

5. F. A. Golightly, F. H. Stott and G. C. Wood, Oxidation of Metals 10, 163 (1976).

6. F. A. Golightly, G. C. Wood and F. H. Stott, Oxidation of Metals 4, 217 (1980).

7. J. Stringer, Materials Science and Engineering: A 120-121, 129 (1989).

8. D. Naumenko, B. A. Pint and W. J. Quadakkers, Oxidation of Metals 86, 1 (2016).

9. V. Kochubey, D. Naumenko, E. Wessel, et al., Materials Letters 60, 1654 (2006).

10. G. J. Tatlock, H. Al-Badairy, R. Bachorczyk-Nagy and R. Fordham, Materials and Corrosion 56, 867 (2005).

11. J. L. Smialek, Surface and Interface Analysis 31, 582 (2001).

12. J. L. Smialek, Metallurgical Transactions A 22, 739 (1991).

13. D. R. Sigler, Oxidation of Metals 32, 337 (1989).

14. D. J. Young, D. Naumenko, L. Niewolak, E. Wessel, L. Singheiser and W. J. Quadakkers, Materials and Corrosion 61, 838 (2010).

15. C. Geers and I. Panas, Oxidation of Metals 91, 55 (2019).

16. V. Babic, C. Geers, B. Jönsson and I. Panas, Electrocatalysis 8, 565 (2017).

17. R. Swadźba, L. Swadźba, J. Wiedermann, M. Hetmańczyk and B. Witala, Oxidation of Metals 82, 195 (2014).

18. Y. Chen, R. C. Reed and E. A. Marquis, Oxidation of Metals 82, 457 (2014).

19. K. A. Unocic and B. A. Pint, Surface and Coatings Technology 237, 8 (2013).

20. K. A. Unocic, E. Essuman, S. Dryepondt and B. A. Pint, Materials at High Temperatures 29, 171 (2012).

21. V. Kochubey, H. Al-Badairy, G. Tatlock, J. Le-Coze, D. Naumenko and W. J. Quadakkers, Materials and Corrosion 56, 848 (2005).

22. D. Naumenko, V. Kochubey, J. Le-Coze, E. Wessel, L. Singheiser and W. J. Quadakkers, Materials Science Forum 461-464, 489 (2004).

23. B. A. Pint, K. L. More and I. G. Wright, Materials at High Temperatures 20, 375 (2003).

24. P. Y. Hou, Journal of the American Ceramic Society 86, 660 (2003).

25. B. A. Pint, A. J. Garratt-Reed and L. W. Hobbs, Journal of the American Ceramic Society 81, 305 (1998). 
26. K. A. Unocic, Y. Chen, D. Shin, B. A. Pint and E. A. Marquis, Micron 109, 41 (2018).

27. V. K. Tolpygo and D. R. Clarke, Materials at High Temperatures 20, 261 (2003).

28. B. A. Pint and K. B. Alexander, Journal of The Electrochemical Society 145, 1819 (1998).

29. B. A. Pint, Oxidation of Metals 45, 1 (1996).

30. B. A. Pint, J. R. Martin and L. W. Hobbs, Oxidation of Metals 39, 167 (1993).

31. A. H. Heuer, D. B. Hovis, J. L. Smialek and B. Gleeson, Journal of the American Ceramic Society 94, 146 (2011).

32. A. H. Heuer, T. Nakagawa, M. Z. Azar, et al., Acta Materialia 61, 6670 (2013).

33. H. Guhl, H.-S. Lee, P. Tangney, et al., Acta Materialia 99, 16 (2015).

34. A. H. Heuer, M. Zahiri Azar, H. Guhl, et al., Journal of the American Ceramic Society 99, 733 (2016).

35. B. A. Pint, Journal of the American Ceramic Society 86, 686 (2003).

36. P. Kofstad, A. Rahmel, R. A. Rapp and D. L. Douglas, Oxidation of Metals 32, 125 (1989).

37. P. Kofstad, High Temperature Corrosion, (Elsevier, Amsterdam, 1988).

38. N. Mortazavi, C. Geers, M. Esmaily, et al., Nature Materials 17, 610 (2018).

39. H. Yu, S. Ukai, S. Hayashi and N. H. Oono, Corrosion Science 127, 147 (2017).

40. MS. Materials Studio 6.0 Accelry Inc.

41. H. J. Monkhorst and J. D. Pack, Physical Review B 13, 5188 (1976).

42. S. Grimme, J. Antony, S. Ehrlich and H. Krieg, The Journal of Chemical Physics 132, 154104 (2010).

43. D. L. Ram, G. J. Tatlock and U. Falke, Materials at High Temperatures 22, 497 (2005).

44. J. T. Ellingham, Journal of the Society of Chemical Industry 63, 125 (1944).

45. G. Brillant, Progress in Nuclear Energy 53, 125 (2011).

46. Y. Yamamoto, B. A. Pint, K. A. Terrani, K. G. Field, Y. Yang and L. L. Snead, Journal of Nuclear Materials 467, 703 (2015).

Publisher's Note Springer Nature remains neutral with regard to jurisdictional claims in published maps and institutional affiliations. 